\title{
Deep Learning Approach for Image Denoising and Image Demosaicing
}

\author{
V. N. V. Satya Prakash \\ Research Scholar \\ JNTUK University, Kakinada \\ Andhra Pradesh, India
}

\author{
K. Satya Prasad, PhD \\ Professor, Dept. of ECE \\ JNTUKU Engineering College \\ Kakinada, A.P., India
}

\author{
T. JayaChandra Prasad, \\ PhD \\ Principal, RGMCET \\ Nandyal, Kurnool \\ Andhra Pradesh, India
}

\begin{abstract}
Color image normally contain of three main colors at the each pixel, but the digital cameras capture only one color at each pixel using color filter array (CFA). While through capturing in color image, some noise/artifacts is added. So, the both demosaicing and de-noising are the first essential task in digital camera. Here, both the technique can be solve sequentially and independently. A conventional neural network based de-noising technique has applied for the removal of noise/artifacts. Afterwards, frequency based demosaicing with the convolutional neural network based image reconstruction algorithm is apply to acquire another two missing color component. The result analysis presented in this paper demonstrate that our proposed de-nosing and demosaicing exhibits the better performance and it is applicable for a large variety of images.
\end{abstract}

\section{General Terms}

Image Reconstruction, Image Demosaicing, Single sensor Array, Demosaicing.

\section{Keywords}

Demosaicing, Color image, Color filter array (CFA), Digital camera, Conventional neural network (CNN)

\section{INTRODUCTION}

There are three main components of the color image by that a natural 'color image' is composed, so it is necessary to capture all three colors by digital camera. For that, we need a camera, which is of at least three sensors. However, the placing of sensor in digital camera is a non-simple problem; furthermore, it requires more space and three sensors cause makes camera to be expensive. Bayer [1] proposed a most simple solution for this problem: a CFA (Color Filter Array). The CFA is placed in front of the sensor, since that the each pixel capture a color component luminance-value (e.g., red, blue 'or' green). While other two-color component should reconstructed to acquire the full "RGB" color image. This process has popularly known as demosaicing.

Demosaicing and de-noising both are the first crucial steps of the digital camera. The existing data of color image (CI) corrupted with the noise and minimum two-third of CI data is missing. For modularity, de-noising and demosaicing are also can be solved sequentially and independently. De-noising suffers from variable per pixel and non-linear noise that introduced via demosaicing. Demosaicing need to handle with unreliable samples that unfortunately causes the error accumulation.

Demosaicing model can be classify as the spatial domain [2], [3], [4] based and frequency domain based [5] and [6]. The various demosaicing techniques detailed survey has presented in the paper [7] and [8]. The literature, shows that the existing 'state-of-art' in demosaicing algorithms bring artifacts in the reassembled image [7], [3], [8], [9]. The goal of de-noising CI is to recover an A image from the noisy $\mathrm{B}$ observation, which is followed by $B=A+W$ degradation model. Commonly $\mathrm{W}$ is additive Gaussian noise (AGN) with the $\sigma$ standard deviation. In image de-noising, image prior modeling plays an important role. Earlier, numerous models has been failed for modeling the image priors, which include nonlocal-selfsimilarity ('NSS') models [1], [10], [11], gradient models [12], [13], and Markov-random-field ('MRF') models [14], [15], [16]. Demand of high de-noising quality, many methodologies of de-noising suffers from the two major drawbacks. First one is, most of the de-noising methodologies involves problem in complex optimization in testing stage, which causes more time in de-noising process [17], [18]. Hence, most of methodologies can rarely achieve the high performance short of sacrificing the computational efficiency. Second is, general models are non-convex and include some manually chosen parameters, in which some providing freedom to increase de-noising performance. A number of discriminative-learning methods have been develop to study the image prior approach. Chen et al. [19], [20] proposed a TNRD (Trainable-nonlinear-reaction diffusion) model that learns an expert modified fields [14] of image prior via unfolding the fixed no. of gradient descent (GD) inference steps. In which the capturing full features of the image structures is limited and, they train only specific model for given noise level.

In this paper, a "frequency domain" based demosaicing methodologies is proposed. According to the frequency component, the chrominance and luminance information is use for reconstructing the image. Firstly, separation of the chrominance and luminance components of the input color image. Afterwards, by the use of 'bilinear interpolation', the three channel of chrominance and a luminance channel has combined to generate an inter-mediate image, and CNN (convolutional neural network) has performed to get the reconstructed image, but somehow image exhibits some artifacts or errors. Hence first, the de-noising model is performed to get more CPSNR. Here, without of doing learning discriminative method with the explicit prior image; we propose an image de-noising as a normal discriminativelearning problem, i.e., sorting out the noise from the noisy image by 'CNN'. The motto for using $\mathrm{CNN}$ in both demosaicing and de-noising is to increase the de-noising performance, optimized CI demosaicing and improve the speed of training process 


\section{RELATED WORK}

Earlier studies also dealing with the problem of demosaicing, several alternative has proposed on basis of image prior. [21] Uses the spectral features of correlation between the color bands and natural images to describe a priority above the image population. Taking account of this approach, the priority image function is independent and can be estimate for all original images. The adaptive prior approach has suggested by the keren et al. [22], which location-based changes in image has based upon the available partial information. Better performance can achieved through reconstructing the G firstly that trailed by the B and $\mathrm{R}$ reconstruction, [23] and [24]. In this type of algorithm, the correlations of inter-color has generally oppressed by the interpolating differences of B-G and R-G. However, there has no optimization performed using these types of differences that means it is not best approach to accomplish the task efficiently. Iterative techniques [25] and vector CFA demosaicing [26], the color transformation approach is proposed by E. Gershikov et al. [27] to optimize color transformation for the 'image demosaicing'.

Residual interpolation for demosaicing algorithm has proposed [28] [29]. In a residual field the interpolation is performs by RI, where the residually described as differences between the observed pixel value and estimated pixel value. Y. Monno et al. [30] proposed an adaptive residual interpolation ("ARI") for the color image demosaicing with Bayer CFA. The ARI selects the suitable number of iteration and add two different RI algorithms, i.e., the MLRI [28] and RI, at every pixels. Further ARI can be improve for the multispectral demosaicing [31].

T. $\mathrm{Yu}$ and $\mathrm{W} . \mathrm{Hu}$ [32] proposed intra Joint inter and channel information for color image demosaicing. This approach exploits the channel color correlation by handling demosaicing problem via a TV(Total variation)-norm optimization. Afterwards, this correlation technique is introduce into the demosaicing framework with using color differences. In [33], $\mathrm{Wu}$ and Timofte proposed an affective post-processing step, which combined with all previously mentioned methodologies of demosaicing, and improve their performance. Generally, it combines with fast ones, so that to get a high-speed performance. The performance of proposed approach can be boost via a post-processing method.

The RI is widely used for 'color image demosaicing' (CID), which is alternative of color difference 'interpolation'. D. Noisy Image
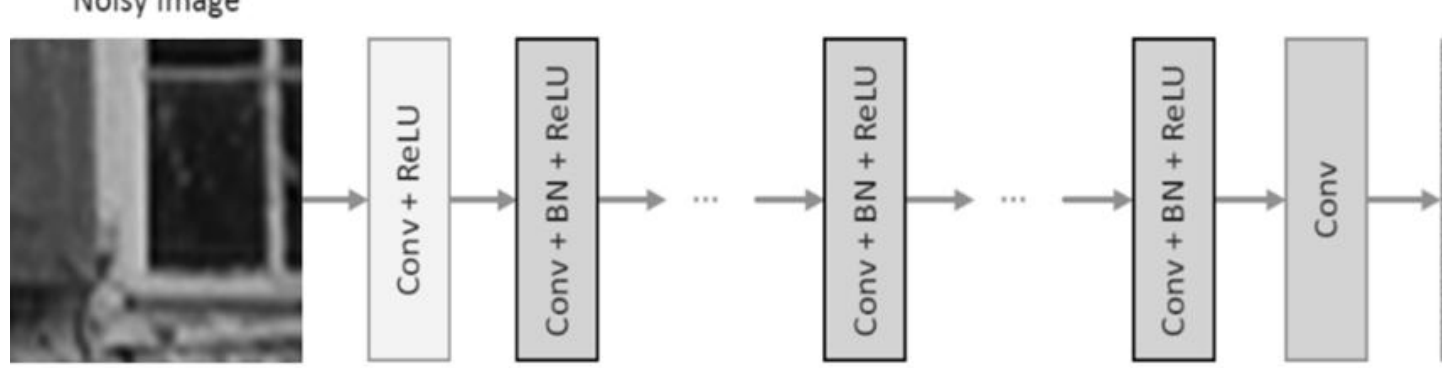

Residual Image

Input to DCNN is the noisy observation $B=A+W$ Denoising discriminative prototypes such as CSF [17] and MLP [31] aim towards study of mapping function $F(B)=A$ to estimate the latent-clean image. Considering DCNN, we approach the residual learning function for training a residual mapping $R(B) \approx W$, afterwards, we have $B=A+R(B)$. The average-mean-square error between estimated and desired residual image from noisy input is consider as the loss function that to study the trainable parameters $\partial$ in DCNN.

$$
L(\partial)=\frac{1}{2 N} \sum_{i=1}^{N}\left\|R\left(B_{i} ; \partial\right)-\left(B_{i}-A_{i}\right)\right\|_{F}^{2}
$$

Here, $\left\{\left(B_{i}, A_{i}\right)\right\}_{i=1}^{N}$ represents N noise-clean training image patch.

Figure 1: The architecture of the Proposed DCNN network

Deep architecture involves DCNN with depth $d$, there are three layer types, which shown in Fig: 1 with the three different color. (i) $c o n v+\operatorname{Re} L U(R L U)$ : in first layer,

$3 \times 3 \times k$ size of filters are used to develop 64 feature maps and the Rectified-Linear-Units (RLU) are then used for nonlinearity. The $\mathrm{k}$ represents no. of image channels, $k=1$ using for gray image and for color image $k=3$. (ii) conv $+B N+\operatorname{ReLU}(R L U)$ :for the layer

2: $(d-1), 64$ filters that of size $3 \times 3 \times 64$ are used and, batch normalization (BN) [28] is added in between RLU and convolution (Conv). (iii) Conv: at last layer, $k$ filters of the size $3 \times 3 \times 64$ has used for reconstruction of output 
For addition, the DCNN model is having two leading features: the adoption of residual learning [29] help to learn $R(B)$ and the batch-normalization [28] process has incorporated to increase the training speed that also maximize the performance of de-noising. Through integrating convolution with RLU and DCNN can progressively separate the structure of image from a noisy one by the hidden layers.

\subsection{Frequency Domain Approach for Demosaicing}

Frequency domain (FD) based approach have consider reconstructing the color image. We consider the luminance component, which extracted in FD to re-construct the original color image using CNN. The proposed image demosaicing model considering the information of luminance in the FD, the steps are as follows.

Alignment of image: using the low frequency information (luminance) set of the images of the CFA Fourier transform images are aligned pairwise.

1. Chrominance and Luminance separation: the input image has extracted in luminance channel and threechrominance channel. The adoption of bilinear interpolation helps to recombine the 3-chrominance and a luminance channel, which result an intermediate image.

2. Reconstruction of image: CNN algorithm has applied to reconstruct the image, which has performed after the bilinear interpolation. Duration the training phase, the approach is done with the both original patches $\mathrm{V}_{\mathrm{i}}$ and intermediate image patches $U_{i}$. After training, the final reconstructed image is obtained.

\subsubsection{Luminance/Chrominance Separation}

In order to interpolate, we separate the luminance and chrominance information, each of the images interpolate separately. The luminance signal has extracted from the CFA images using a low-pass filter $\mathrm{F}$ identified by Alleyson et al [39] (Eqn. (2)). By subtracting the luminance information from the red, green and blue channels of the CFA image and demodulating the result, the three-chrominance parts (for red, green and blue) has obtained. In a luminance, image this result $\alpha$ and three chrominance images $S_{1}, S_{2}$ and $S_{3}$ all at the original image size.

$$
\begin{aligned}
& \alpha=\mathrm{I}_{\mathrm{CFA}} * \mathrm{~F} \\
& \mathrm{~S}_{1}=\left(\left(\mathrm{I}_{\mathrm{CFA}}-\alpha\right) \text { e } \mathrm{T}_{1}\right) * \mathrm{R}_{1} \\
& \mathrm{~S}_{2}=\left(\left(\mathrm{I}_{\mathrm{CFA}}-\alpha\right) \text { e } \mathrm{T}_{2}\right) * \mathrm{R}_{2} \\
& \mathrm{~S}_{3}=\left(\left(\mathrm{I}_{\mathrm{CFA}}-\alpha\right) \text { e } \mathrm{T}_{3}\right) * \mathrm{R}_{1} \\
& \text { With } \mathrm{R}_{1}=1 / 4\left(\begin{array}{ccc}
1 & 2 & 1 \\
2 & 4 & 2 \\
1 & 2 & 1
\end{array}\right) \text { and } \mathrm{R}_{2}=1 / 4\left(\begin{array}{lll}
0 & 1 & 0 \\
1 & 4 & 1 \\
0 & 1 & 0
\end{array}\right)
\end{aligned}
$$

The matrices $R_{1}$ and $R_{2}$ given above are two demodulation (or interpolation) filters, and the symbol $\mathrm{e}$ is used represent a point wise multiplication of two matrices. The $T_{1}$,
$\mathrm{T}_{2}$ and $\mathrm{T}_{3}$ are modulation matrices for each of the chrominance parts. The process of computing their mass resolution versions using Normalized Convolution (NC) approach proposed in [40] on each of the four channel separately. To have the highest contributions from samples close to the considered pixel, a Gaussian weighting function (applicability function) is used and they make use of a variance $\sigma^{2}=2$.

The computing process of a pixel of the high-resolution image has done from the pixels in a neighbour-hood around, it as:

$$
\mathrm{U}^{\prime}=\left(\mathrm{b}^{\mathrm{T}} \mathrm{Wb}\right)^{-1} \mathrm{~b}^{\mathrm{T}} \mathrm{Wf}
$$

where $\mathrm{f}$ is said to be $\mathrm{N} \times 1$ vector which contains the 'neighbour-hood pixels', $\mathrm{b}$ is an $\mathrm{N} \times \mathrm{m}$ matrix of $\mathrm{m}$ basis functions tested at the local coordinates of the pixels $f$, and $\mathrm{W}$ is an $\mathrm{N} \times \mathrm{N}$ weighting matrix at the pixel coordinates which contains the Gaussian weights sampled. The interpolated pixel value has given by the first element of the $m \times 1$ vector $U^{\prime}$. A circular region with radius four times the pixel distance of the high-resolution image has used for neighbourhood selection. The pixels number $\mathrm{N}$ in this region may differ depending on the location because of the nonuniform grid.

For the luminance channel $\alpha$ we perform bilinear interpolation, as well as for each of the chrominance channels $\mathrm{S}_{1}, \mathrm{~S}_{2}$ and then we add luminance and chrominance together, which results in an intermediate high-resolution colour image $\mathrm{U}$. To compute the $\mathrm{U}$, we consider $\mathrm{N}$ pixels of the highresolution image can be given as

$$
\mathrm{U}=\sum \sum \mathrm{U}^{\prime}
$$

Here $U$ give rise to error / artifacts, to compute high resolution image, the final high resolution image are computed by fitting a polynomial surface and in the quality of interpolation such as using Low-order NC with a large applicability window cannot reconstruct small details in the image, it is a scale of the applicability function which plays a decisive role.

\subsubsection{Image Reconstruction}

We assume $U$ is the intermediate image, which has obtained from Eqn.(4) and $\mathrm{V}$ is the perfect demosaiced image; we formulate the image corruption process below

$$
\mathrm{U}=\Omega(\mathrm{V})
$$

Where the value of $\Omega=R^{n} \rightarrow R^{n}$ which is an arbitrary stochastic error process induced because of the polynomial surface fitting. Later, demosaicing learning objective becomes:

$$
\mathrm{g}=\underset{\mathrm{g}}{\operatorname{argmin}} \mathrm{A}_{\mathrm{Q}}\|\mathrm{g}(\mathrm{U})-\mathrm{V}\|_{2}^{2}
$$

In Eqn. (6), the task is to find a function ' $\mathrm{g}$ ' that best approximates $\Omega^{-1}$. By choosing appropriate $\Omega$ in different situations, we can treat the image demosaicing problem in a unified framework. 


\subsubsection{Image Reconstruction Demosaicing Auto} Encoder (IRDA)

Let $V_{i}$ be the original data for $i=1,2,3 \ldots . . N$, where $U_{i}$ be the corrupted version of corresponding $\mathrm{V}_{\mathrm{i}}$

$$
\begin{aligned}
& \mathrm{h}\left(\mathrm{U}_{\mathrm{i}}\right)=\varphi\left(\mathrm{W}_{\mathrm{P}_{\mathrm{i}}}+\mathrm{b}\right) \\
& W^{\prime}\left(\mathrm{U}_{\mathrm{i}}\right)=\varphi\left(\mathrm{W}^{\prime} \mathrm{h}\left(\mathrm{U}_{\mathrm{i}}\right)+\mathrm{b}^{\prime}\right)
\end{aligned}
$$

Where $\varphi\left(\mathrm{U}_{\mathrm{i}}\right)=(1+\exp (-\mathrm{U}))^{-1}$ is known as sigmoid activation function, which is applied component wise to vectors, hidden layer activation is $h_{i}, \quad \forall\left(U_{i}\right)$ is an approximation of $\mathrm{V}_{\mathrm{i}}$ and the weights and biases is represented by $\omega=\left\{W, b, W^{\prime}, b^{\prime}\right\}$. To minimize the reconstruction loss, IRDA can trained with various optimization methods

$$
\omega=\underset{\omega}{\operatorname{argmin}} \sum_{i=1}^{\mathrm{N}}\left\|\mathrm{V}_{\mathrm{i}}-\mathbb{W}^{\prime}\left(\mathrm{U}_{\mathrm{i}}\right)\right\|
$$

After training IRDA, by using the hidden layer activation of the first layer as the input of the next layer, we move on to training the next layer.

\subsubsection{Convolution Neural Network Based Image Reconstruction Algorithm}

In this section, the proposed model Convolutional Neural Network Based Image Reconstruction Algorithms structure and optimization objective has described. The entire image is intractable due to direct processing; we draw overlapping patches instead from the image as our data objects.

In the training phase, with both the corrupted error image patches, $\mathrm{U}_{\mathrm{i}}$ the model is supply, for $\mathrm{i}=1,2,3 \ldots \mathrm{N}$ and the original patches $\mathrm{V}_{\mathrm{i}}$. IRDA will be able to reconstruct the corresponding clean image given any error observation only after the training.

To combine sparse coding and neural networks and avoid over-fitting, and to minimize the reconstruction loss regularized by a sparsity-inducing term we train IRDA.

$$
\left.\mathrm{M}_{1}(\mathrm{U}, \mathrm{V}, \omega)=\frac{1}{\mathrm{~N}} \sum_{i=1}^{N} \frac{1}{2}\left\|\mathrm{~V}_{\mathrm{i}}-\mathrm{W}^{\mathrm{W}}(\mathrm{U})\right\|_{2}^{2}+\delta \mathrm{KL}(\$) \Gamma\right)+\frac{\lambda}{2}\left(\|\mathrm{~W}\|_{\mathrm{S}}^{2}+\left\|\mathrm{W}^{\prime}\right\|_{\mathrm{S}}^{2}\right)
$$

$$
\begin{aligned}
& \mathrm{KL}(\hat{\Gamma} \| \Gamma)=\sum_{j=1}^{|\hat{\Gamma}|} \Gamma \log \frac{\Gamma}{\hat{\Gamma}_{j}}+(1-\Gamma) \log \frac{(1-\Gamma)}{1-\hat{\Gamma}} \\
& \hat{\Gamma}=\frac{1}{\mathrm{~N}} \sum_{i}^{N} \mathrm{~h}\left(\mathrm{U}_{\mathrm{i}}\right)
\end{aligned}
$$

Where $\mathrm{h}($.$) and \hat{\mathrm{V}}($.$) are explained in Eqn. (7) and Eqn.$ (8) respectively. Here the average activation of the hidden layer is $\hat{\Gamma}$. By choosing small, $\Gamma$ we regularize the hidden layer representation to be sparse, so that the mean activation of hidden units to be small has been encouraged by the KM divergence term. Therefore, most of the time the hidden units will be zero and achieve sparsity.

We use $h\left(V_{i}\right)$ and $h\left(U_{i}\right)$ as the clean and error input respectively for the second IRDA only after training of the first IRDA. Since in a different space from $V_{i}$ the $h\left(V_{i}\right)$ lies, the meaning of applying $\Omega($.$) to h\left(\mathrm{~V}_{\mathrm{i}}\right)$ is not so clear. We used $\Omega\left(h\left(V_{i}\right)\right)$ as the error input after discarding $U_{i}$.

With the weights obtained from $\mathrm{K}$ layered IRDAs, we initialize a deep network. In which a network has one input layer, one output and $2 \mathrm{~K}-1$ hidden layers. Using the standard back-propagation algorithm the entire network has trained to minimize the below objective:

$$
\mathrm{M}_{2}(\mathrm{U}, \mathrm{V}, \omega)=\frac{1}{\mathrm{~N}} \sum_{i=1}^{N} \frac{1}{2}\left\|\mathrm{~V}_{\mathrm{i}}-\mathrm{V}^{\mathrm{V}}(\mathrm{U})\right\|_{2}^{2}+\frac{\lambda}{2} \sum_{j=1}^{2 \mathrm{~K}}\left(\left\|\mathrm{~W}_{\mathrm{j}}\right\|_{\mathrm{s}}^{2}\right)
$$

Because of pre-trained weights will serve as regularization to the network, we removed the sparsity regularization [41], according to [42], the loss functions are optimized with LBFGS algorithm to achieve fastest convergence in our settings is done during pre-training and fine tuning stages.

\section{RESULT ANALYSIS}

This section deals with the achieved results by applying our proposed methodologies for the color image de-noising and demosaicing. This approach is implemented using 2016b MATLAB version tool on the dataset, which are publicly available. For the evaluation of performance, we considering $24 \mathrm{bmp}$ images from Kodak dataset that is of $768 \times 512$ in size. In our proposed approach each of the image is de-noises with the CNN and Afterwards, Frequency domain Approach is apply for demosaicing that is followed by CNN. This proposed approach compared with the other modeled technologies and the quality of performance is compute in terms of PSNR value of Green, Red and Blue channels. Figure 2 shows the 24 Kodak images that we are considering as a dataset.

For calculating the performance of our proposed methodologies, we differentiate the output demosaiced image and the original image and calculate its CPSNR ("color-peak signal-to-noise ratio"). For the each channel of every Kodak image, separate CPSNR has calculated and the CPSNR of reconstructed image. The CPSNR value of combined channel is calculate as:

$$
\mathrm{CPSNR}=10 \log \frac{(255)^{2}}{\frac{1}{3 \times \mathrm{H} \times \mathrm{W}} \sum_{i}^{3} \sum_{x=0}^{\mathrm{H}-1} \sum_{y=0}^{\mathrm{W}-1}\left(I_{O}(x, y, i)-I_{d}(x, y, i)^{2}\right.}
$$

Here, $I_{O}$ is the original input image; $\mathrm{H}$ denotes height of image, $\mathrm{W}$ width of image and $I_{d}$ is the demosaiced image. The value of ' $i$ ' varies from 1 to 3 based on three color planes. 


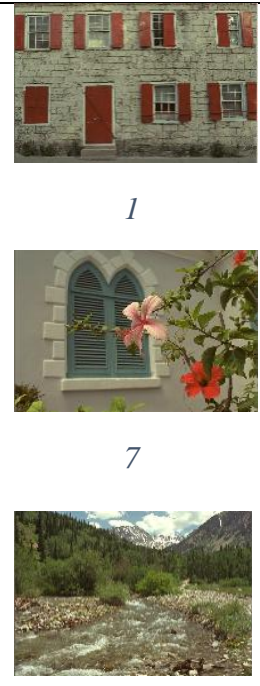

13

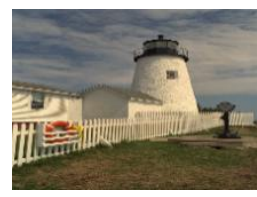

19

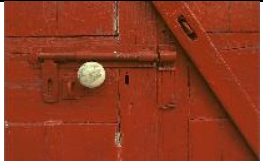

2

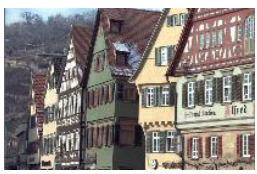

8

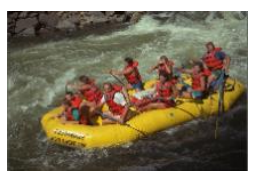

14

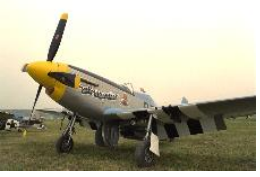

20

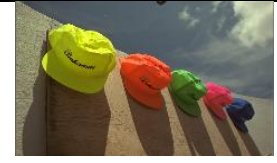

3

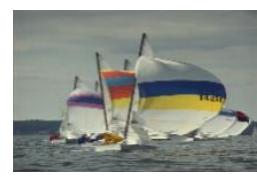

9

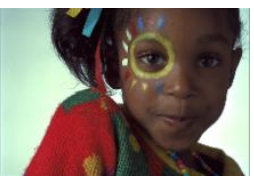

15

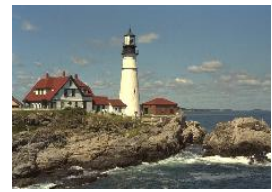

21

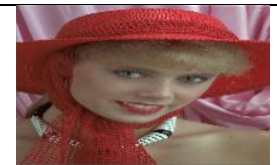

4

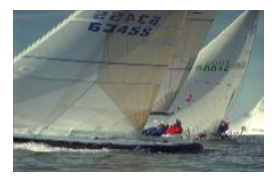

10

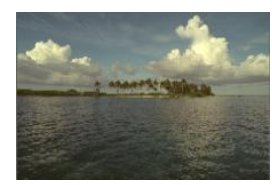

16

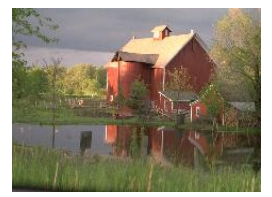

22

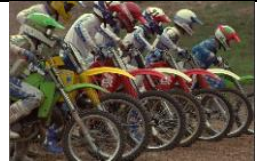

5

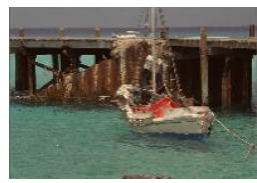

11

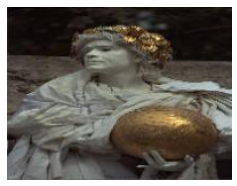

17

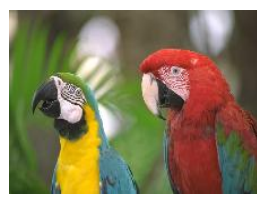

23

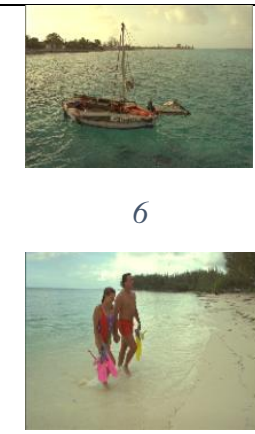

12

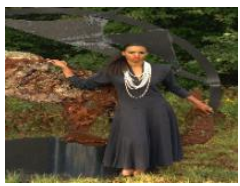

18

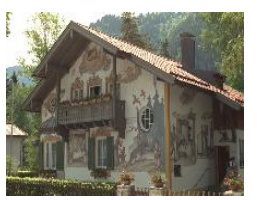

24

Figure 2: Kodak Dataset Consisting of 24 images

Table 1: De-noising and Demosaicing PSNR Performance values

\begin{tabular}{|c|c|c|c|c|c|c|c|c|}
\hline & \multicolumn{4}{|c|}{ De-noising } & \multicolumn{4}{c|}{ Demosaicing } \\
\hline $\begin{array}{c}\text { Image } \\
\text { no. }\end{array}$ & $\begin{array}{c}\text { Red } \\
\text { channel }\end{array}$ & $\begin{array}{c}\text { Green } \\
\text { channel }\end{array}$ & $\begin{array}{c}\text { Blue } \\
\text { channel }\end{array}$ & CPSNR & $\begin{array}{c}\text { Red } \\
\text { channel }\end{array}$ & $\begin{array}{c}\text { Green } \\
\text { channel }\end{array}$ & $\begin{array}{c}\text { Blue } \\
\text { channel }\end{array}$ & CPSNR \\
\hline 1 & 37.7354 & 41.4675 & 38.2405 & 38.8637 & 45.4655 & 47.2035 & 45.3736 & 45.8887 \\
\hline 2 & 39.0606 & 45.1107 & 42.5118 & 41.5272 & 48.0027 & 50.5756 & 48.4814 & 48.8034 \\
\hline 3 & 43.3546 & 46.5302 & 42.5245 & 43.8251 & 49.1246 & 50.9741 & 49.2229 & 49.6462 \\
\hline 4 & 38.3593 & 45.2273 & 43.4255 & 41.3203 & 48.4641 & 50.7808 & 48.8523 & 49.1861 \\
\hline 5 & 38.1082 & 41.1222 & 37.2559 & 38.5395 & 46.0416 & 47.6154 & 46.0948 & 46.4884 \\
\hline 6 & 40.2328 & 43.6045 & 39.0661 & 40.582 & 46.0330 & 48.1142 & 46.0370 & 46.5609 \\
\hline 7 & 43.0347 & 46.1126 & 42.2135 & 43.4916 & 49.2400 & 50.9804 & 49.1835 & 49.6781 \\
\hline 8 & 35.7171 & 40.0911 & 35.7421 & 36.7601 & 44.8327 & 46.9167 & 44.8485 & 45.3660 \\
\hline 9 & 42.5682 & 45.8929 & 42.5371 & 43.4085 & 48.7392 & 50.8552 & 49.1579 & 49.4353 \\
\hline 10 & 42.0079 & 45.8017 & 42.2295 & 43.0358 & 48.8379 & 50.9301 & 49.1515 & 49.4899 \\
\hline 11 & 39.4112 & 43.2493 & 40.1635 & 40.6525 & 46.7951 & 48.7441 & 46.9064 & 47.3413 \\
\hline 12 & 43.3349 & 47.2484 & 43.4017 & 44.3206 & 48.9786 & 51.0344 & 48.8698 & 49.4553 \\
\hline 13 & 34.722 & 37.4754 & 33.5855 & 34.976 & 44.3970 & 45.6242 & 44.3169 & 44.7141 \\
\hline 14 & 36.9257 & 41.1428 & 37.1498 & 38.0263 & 46.4603 & 48.3953 & 46.7689 & 47.0798 \\
\hline 15 & 37.8368 & 43.3049 & 41.0215 & 40.1424 & 48.3634 & 50.1556 & 48.4517 & 48.8695 \\
\hline 16 & 43.674 & 46.8584 & 42.9165 & 44.1786 & 47.3418 & 49.6829 & 47.3572 & 47.9200 \\
\hline 17 & 41.4636 & 43.9943 & 40.7508 & 41.8624 & 48.5354 & 50.1183 & 48.6040 & 48.9901 \\
\hline 18 & 36.6123 & 39.6548 & 36.6678 & 37.4327 & 46.2079 & 47.5695 & 46.3231 & 46.6310 \\
\hline 19 & 40.1549 & 43.7405 & 40.6933 & 41.2687 & 47.1127 & 49.1092 & 47.3704 & 47.7250 \\
\hline 20 & 42.512 & 45.0649 & 39.7337 & 41.9031 & 48.6013 & 50.2336 & 48.6583 & 49.0621 \\
\hline 21 & 39.5372 & 42.6231 & 38.0802 & 39.6994 & 46.6647 & 48.4007 & 46.6422 & 47.1156 \\
\hline
\end{tabular}




\begin{tabular}{|c|c|c|c|c|c|c|c|c|}
\hline 22 & 38.7829 & 41.5843 & 38.0229 & 39.215 & 47.4979 & 49.0188 & 47.3288 & 47.8450 \\
\hline 23 & 42.946 & 46.4835 & 43.2831 & 43.9731 & 49.9882 & 51.9456 & 50.6982 & 50.7561 \\
\hline 24 & 35.8236 & 38.0721 & 33.0117 & 35.146 & 46.2462 & 47.5865 & 45.9628 & 46.5076 \\
\hline Average & $\mathbf{3 9 . 7 4 6 5}$ & $\mathbf{4 3 . 3 9 4 0}$ & $\mathbf{3 9 . 7 5 9 5}$ & $\mathbf{4 0 . 5 8 9 6}$ & $\mathbf{4 7 . 4 1 5 5}$ & $\mathbf{4 9 . 2 7 3 6}$ & $\mathbf{4 7 . 5 2 7 6}$ & $\mathbf{4 7 . 9 3 9 8}$ \\
\hline
\end{tabular}

Table 1 represents the additional information of denoising and, R, G, and B channels PSNR. According to the results, it seems that our proposed denoising and demosaicing methodologies is effective and robust on the wide range of Kodak dataset images. The average CPSNR value of (proposed) de-noised 24 Kodak dataset image is $40.59 \mathrm{~dB}$ and for the demosaicing case, CPSNR value is $47.94 \mathrm{~dB}$. An image from the Kodak dataset has taken to show the variation in image, original image to de-noise image and afterwards, de-noise image to Demosaic image. Figure 3 shows the

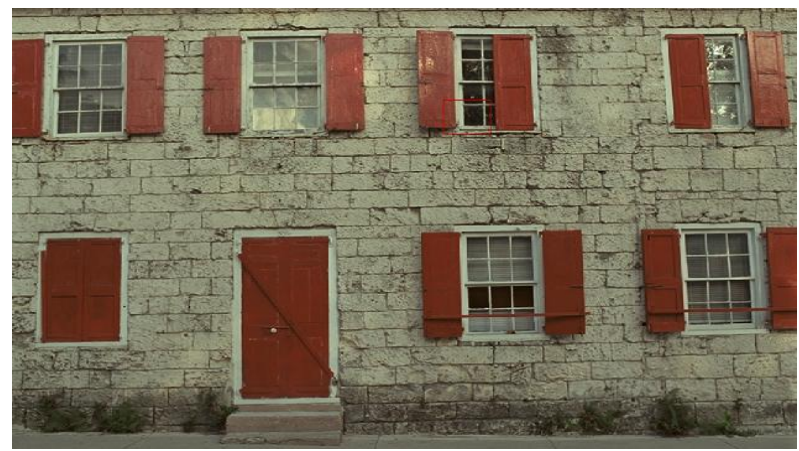

(a)

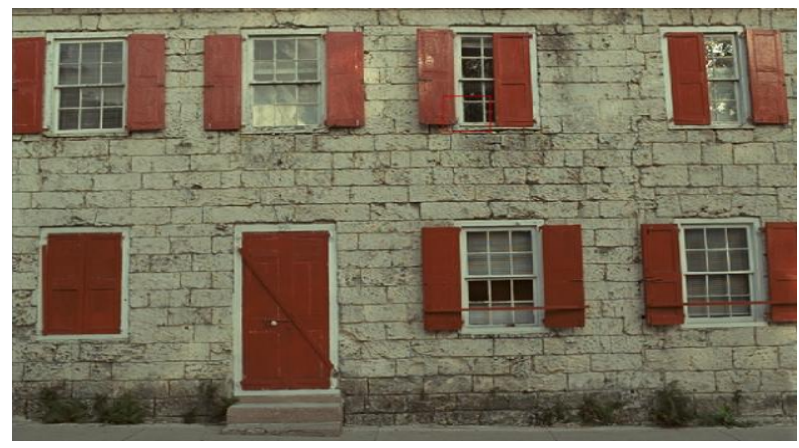

(c)

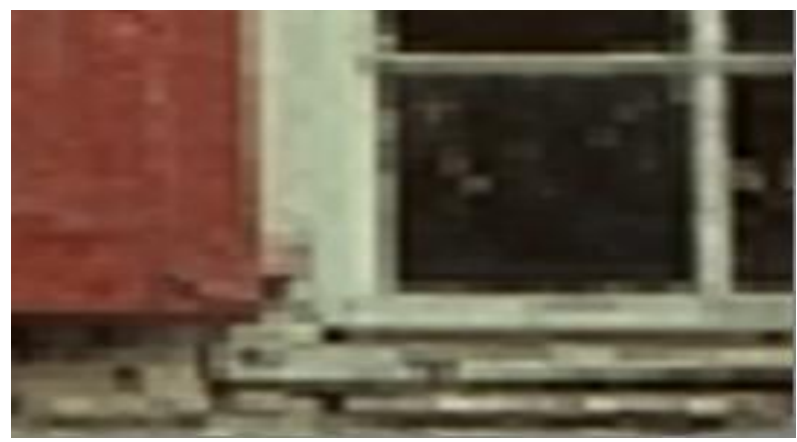

(b) original image, de-noise image and demosaiced image, this is the first image from the Kodak dataset. For the comparison of proposed approach, performance with other existing approaches is meaningful to find out the optimized result. Here, table 2 shows the comparison of the existing approaches and our proposed approach. The average values of 24 Kodak images has taken of Circ4 [43], Wang CFA [44], DDR [33], FR [33], MLRI [28] and our proposed approach CPSNR values.

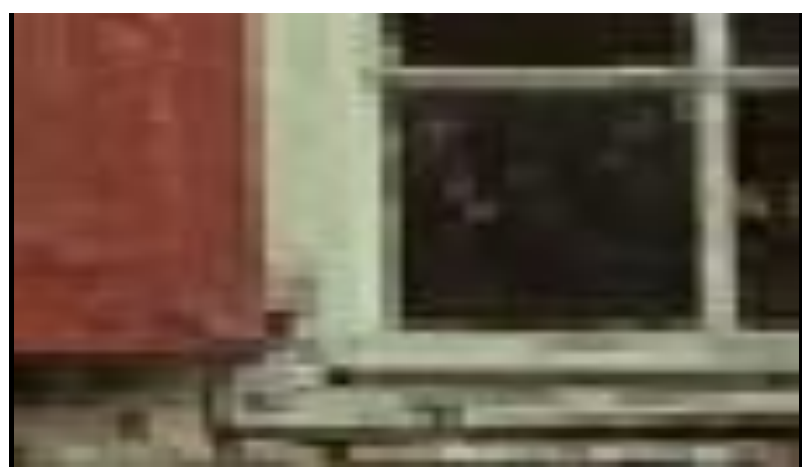

(d)

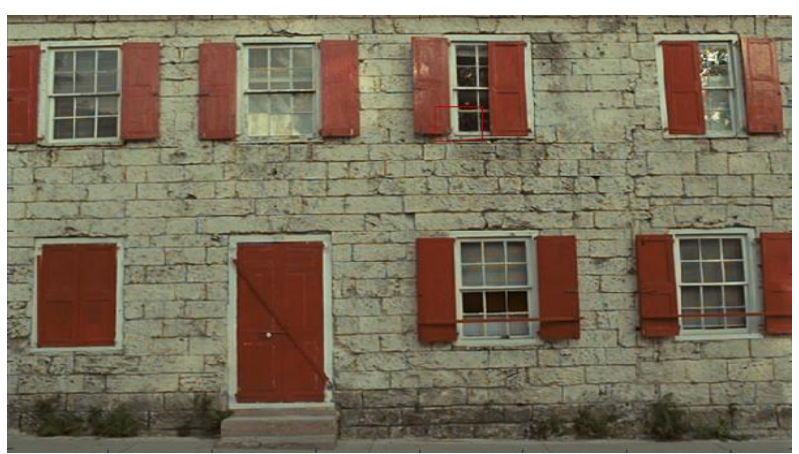

(e)

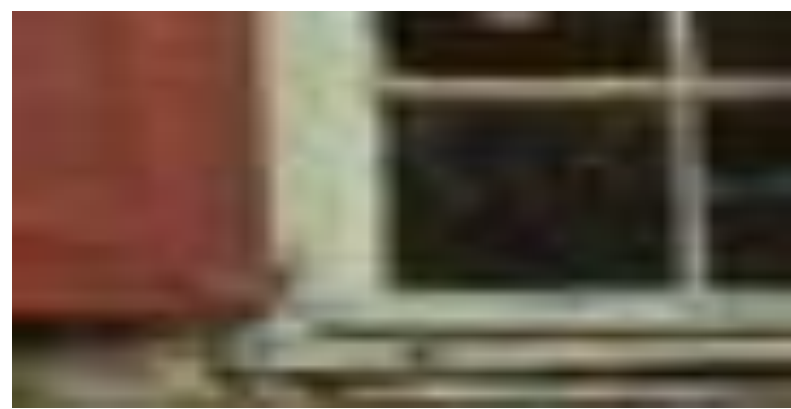

$(f)$

Figure 3: (a) Original image from Kodak dataset, (b) Zoomed red square part of original image, (c) De-noise image,

(d) Zoomed red square part of de-noise image, (e) Demosaic output image and (f) Zoomed red square part of demosaic image. 
Table 2: Comparison table of the existing methods with our proposed approach. (In terms of CPSNR)

\begin{tabular}{|c|c|c|c|c|c|c|}
\hline Image No. & DDR[33] & FR[33] & Circ4[42] & Wang CFA[43] & MLRI[28] & Our Proposed Method \\
\hline 1 & 40.0882 & 39.7031 & 39.17 & 35.35 & 43.43 & 45.8888 \\
\hline 2 & 41.4867 & 41.6328 & 39.31 & 38.11 & 40.23 & 48.8035 \\
\hline 3 & 44.057 & 44.1495 & 37.83 & 38.4 & 45.11 & 49.6462 \\
\hline 4 & 41.4366 & 41.5841 & 39.88 & 38.32 & 44.26 & 49.1861 \\
\hline 5 & 39.3056 & 39.3367 & 33.93 & 33.62 & 44.22 & 46.4884 \\
\hline 6 & 41.4361 & 41.326 & 39.33 & 36.74 & 44.52 & 46.5609 \\
\hline 7 & 43.687 & 43.8461 & 37.51 & 38.83 & 44.33 & 49.6781 \\
\hline 8 & 37.5114 & 37.3933 & 36.36 & 33.37 & 37.67 & 45.366 \\
\hline 9 & 43.8016 & 43.7787 & 39.19 & 39.33 & 40.12 & 49.4353 \\
\hline 10 & 43.3092 & 43.3516 & 40.71 & 38.12 & 40.62 & 49.4899 \\
\hline 11 & 41.3479 & 41.2523 & 39.29 & 36.77 & 43.34 & 47.3413 \\
\hline 12 & 44.6544 & 44.7106 & 41.03 & 40.61 & 46.92 & 49.4553 \\
\hline 13 & 36.1702 & 35.788 & 33.7 & 31.75 & 40.43 & 44.7141 \\
\hline 14 & 38.2368 & 38.37 & 33.36 & 33.3 & 43.8 & 47.0798 \\
\hline 15 & 40.1363 & 40.1631 & 38.11 & 37.6 & 45.52 & 48.8695 \\
\hline 16 & 44.772 & 44.7449 & 42.24 & 39.89 & 44.55 & 47.9201 \\
\hline 17 & 42.4419 & 42.4173 & 39.92 & 39.11 & 39.89 & 48.9901 \\
\hline 18 & 38.1835 & 38.0551 & 34.92 & 34.83 & 37.31 & 46.6310 \\
\hline 19 & 42.049 & 41.9158 & 39.46 & 37.63 & 42.69 & 47.7249 \\
\hline 20 & 42.3627 & 42.3495 & 38.3 & 38.02 & 43.11 & 49.0621 \\
\hline 21 & 40.5918 & 40.3939 & 38.23 & 36.69 & 41.45 & 47.1156 \\
\hline 22 & 39.4463 & 39.564 & 36.58 & 36.55 & 42.19 & 47.845 \\
\hline 23 & 44.0747 & 44.2184 & 37.17 & 39.2 & 45.07 & 50.7561 \\
\hline 24 & 35.7649 & 35.6556 & 34.84 & 33.23 & 45.35 & 46.5076 \\
\hline Average & 41.0980 & 41.0708 & 37.9321 & 36.8904 & 42.7554 & 47.9398 \\
\hline
\end{tabular}

Our proposed model (Average CPSNR of 47.94) is compared with Circ4 [42] (Average CPSNR of $37.93 \mathrm{~dB}$ ) and it is $20.87 \%$ less $\mathrm{dB}$ compared to our proposed approach. When ours one compared to Wang CFA [43] (Average CPSNR of $36.9 \mathrm{~dB}), 23.05 \%$ improvement is seems in our proposed CPSNR $14.27 \%$.of improvement is seems, when it compared to DDR [33] that average CPSNR value is $41.09 \mathrm{~dB}$.

Similarly, improved performance has achieved as $14.33 \%$ and $10.81 \%$ when it compared from FR [33] (Average CPSNR of $41.07 \mathrm{~dB}$ ) and MLRI [28] (Average CPSNR of $42.75 \mathrm{~dB}$ ). From the above comparison, it is concluded that our proposed methods performs better compared to the other algorithm for the CI (Color Image) demosaicing

\section{CONCLUSION}

The importance of demosaicing and de-noising is discussed in this paper. The Color image(CI) taken using CFA has corrupted with noise and minimal two-third of CI data is missing. Both de-noising and demosaicing techniques are performed independently and sequentially. Coupling denoising with demosaicing is essential to improve image quality .Very Limited amount of work is carried out by considering de-noising along with demosaicing. The drawbacks of existing systems are clearly discussed in this paper. To overcome these drawbacks the proposed model considers frequency domain approach for demosaicing.
Adaption in demosaicing is achieved using CNN. To perform de-noising, recently introduced $\mathrm{CNN}$ is adopted in the proposed model. Modeling and realization of proposed model is discussed in this paper. The commonly used KODAK dataset is considered for performance evaluation. Comparison results obtained using proposed model and other state of art techniques is presented. Superior performance of proposed model is proved based on results obtained. Coupling denoising with demosaicing improves performance when compared to solitary demosaicing algorithms.

In the future, evaluating performance on additional CI data can be taken into consideration. Exploring additional denoising techniques is another possible direction for future scope.

\section{REFERENCES}

[1] Lu, Y.M.; Karzand, M.; Vetterli, M., "Demosaicking by Alternating Projections: Theory and Fast One-Step Implementation," in IEEE Transactions on Image Processing, vol.19, no.8, pp.2085-2098, Aug. 2010

[2] Z. Zha, X. Liu, X. Huang, X. Hong, H. Shi, Y. Xu, Q. Wang, L. Tang, and X. Zhang, "Analyzing the group sparsity based on the rank minimization methods," arXiv preprint arXiv:1611.08983, 2016 
[3] L. I. Rudin, S. Osher, and E. Fatemi, "Nonlinear total variation based noise removal algorithms," Physica D: Nonlinear Phenomena, vol. 60, no. 1, pp. 259-268, 1992

[4] S. Osher, M. Burger, D. Goldfarb, J. Xu, and W. Yin, "An iterative regularization method for total variationbased image restoration," Multiscale Modeling \& Simulation, vol. 4, no. 2, pp. 460-489, 2005.

[5] J. Xu, L. Zhang, W. Zuo, D. Zhang, and X. Feng, "Patch group based nonlocal self-similarity prior learning for image denoising," in International Conference on Computer Vision, 2015, pp. 244-252.

[6] W. Dong, L. Zhang, G. Shi, and X. Li, "Nonlocally centralized sparse representation for image restoration," IEEE Transactions on Image Processing, vol. 22, no. 4, pp. 1620-1630, 2013.

[7] A.Buades, B. Coll, and J.-M. Morel, "Nonlocal image and movie denoising," International Journal of Computer Vision, vol. 76, no. 2, pp. 123-139, 2008.

[8] Y. Weiss and W. T. Freeman, "What makes a good model of natural images?" in IEEE Conference on Computer Vision and Pattern Recognition, 2007, pp. 1-8

[9] X. Lan, S. Roth, D. Huttenlocher, and M. J. Black, "Efficient belief propagation with learned higher-order Markov random fields," in European Conference on Computer Vision, 2006, pp. 269-282.

[10] Ousman Boukara,Laurent Bitjokaa and Gamraïkréo Djaowéa. "Nondestructive Determination Of Beans Water Absorption Capacity Using CFA Images Analysis For Hard-To-Cook Evaluation". International Journal of Electrical and Computer Engineering (IJECE).Vol. 3, No. 3, June 2013, pp. 317 328

[11] Nai-Xiang Lian; Lanlan Chang; Yap-Peng Tan; Zagorodnov, V., "Adaptive Filtering for Color Filter Array Demosaicking," in IEEE Transactions on Image Processing , vol.16, no.10, pp.2515-2525, Oct. 2007

[12] Xiao Zhou; Fanfan Yang; Chunxiao Zhang; Chengyou Wang, "Improved adaptive demosaicking using directional weighting," in Computer Science \& Education (ICCSE), $20149^{\text {th }}$ International Conference on , vol., no., pp.615-618, 22-24 Aug. 2014

[13] Zhang, D.; Xiaolin Wu, "Color demosaicking via directional linear minimum mean square-error estimation," in IEEE Transactions on Image Processing, vol.14, no.12, pp.2167-2178, Dec. 2005

[14] Kim, Y.; Jeong, J., "Four Direction Residual Interpolation for Demosaicking," in IEEE Transactions on Circuits and Systems for Video Technology, vol.PP, no.99, pp.1-1. doi: 10.1109/TCSVT.2015.2428552

[15] Maschal, R.A.; Young, S.S.; Reynolds, J.P.; Krapels, K.; Fanning, J.; Corbin, T., "New Image Quality Assessment Algorithms for CFA Demosaicing," in IEEE Sensors Journal , vol.13, no.1, pp.371-378, Jan. 2013

[16] Xiangdong Chen; Liwen He; Gwanggil Jeon; Jechang Jeong, "Multidirectional Weighted Interpolation and Refinement Method for Bayer Pattern CFA Demosaicking," in IEEE Transactions on Circuits and Systems for Video Technology, vol.25, no.8, pp.12711282, Aug. 2015
[17] R.Niruban, T.Sree Renga Raja and R.Deepa. "Similarity and Variance of Color Difference Based Demosaicing." TELKOMNIKA Indonesian Journal of Electrical Engineering. Vol. 13, No. 2, February 2015, pp. 238-246

[18] Michael, G.; Kiryati, N., "Example based demosaicing," in 2014 IEEE International Conference on Image Processing (ICIP), vol., no., pp.1832-1836, 27-30 Oct. 2014

[19] Lu, Y.M.; Karzand, M.; Vetterli, M., "Demosaicking by Alternating Projections: Theory and Fast One-Step Implementation," in IEEE Transactions on Image Processing, vol.19, no.8, pp.2085-2098, Aug. 2010

[20] Wei Ye; Kai-Kuang Ma, "Color Image Demosaicing Using Iterative Residual Interpolation," in IEEE Transactions on Image Processing, , vol.24, no.12, pp.5879-5891, Dec. 2015

[21] D.H. Brainard and D. Sherman," Reconstructing images from trichromatic samples: From basic research to practical applications". In Proc. of the IS\&T /SID, 1995.

[22] D. Keren. "An adaptive bayesian approach to demosaicing color images." Technical Report HPL-96129, Hewlett-Packard, 1996.

[23] K.-H. Chung and Y.-H. Chan. "Color demosaicing using variance of color differences." IEEE Trans. on Image Processing 15:2944-2955, 2006.

[24] R. Sher and M. Porat. "CCD Image Demosaicing using Localized Correlations." In Proc. of EUSIPCO, Poznan, Poland, Sept. 2007.

[25] R. Kimmel,'Demosaicing: Image reconstruction from color CCD samples." IEEE Trans. on Image Processing 8:1221-1228, 1999.

[26] M. R. Gupta and T. Chen." Vector color filter array demosaicing." in Proc. of SPIE, Sensors and Camera Systems for Scientific, Industrial, and Digital Photography Applications II 4306:374-382, 2001.

[27] E. Gershikov. "Optimized Color Transforms for Image Demosaicing" International Journal Of Computational Engineering Research (ijceronline.com) Vol. 3 Issue. 3. Mar 2013

[28] D. Kiku, Y. Monno, M. Tanaka, and M. Okutomi, "Minimized-Laplacian residual interpolation for color image demosaicking," Proc. of SPIE, vol. 9023, pp. 90230L-1-8, 2014.

[29] W. Ye and K. K. Ma, "Image demosaicing by using iterative residual interpolation," Proc. of IEEE Int. Conf. on Image Processing (ICIP), pp. 1862-1866, 2014.

[30] Y. Monno and D. Kiku. "Adaptive residual interpolation for color image demosaicking" in IEEE Transactions on Image Processing ,pp.3861-3865,2015

[31] Y. Monno, D. Kiku, S. Kikuchi, M. Tanaka, and M. Okutomi, "Multispectral demosaicking with novel guide image generation and residual interpolation," Proc. of IEEE Int. Conf. on Image Processing (ICIP), pp. 645 649,2014

[32] T. Yu, W. Hu, W. Xue and W. Zhang "Colour image demosaicking via joint intra and inter channel information" electronics letters Vol. 52 No. 8 pp. 605 607. 14th April 2016. 
[33] Jiqing Wu, Radu Timofte, "Demosaicing based on Directional Difference Regression and Efficient Regression Priors" in IEEE Transactions on image Processing, 2016

[34] Daisuke Kiku, Yusuke Monno, "Beyond Color Difference: Residual Interpolation for Color Image Demosaicking" IEEE Transactions on Image Processing, vol. 25, no. 3, March 2016.

[35] I.Pekkucuksen and Y. Altunbasak, "Gradient based threshold free color filter array interpolation," in Proc. of IEEE Int. Conf. Image Process. (ICIP), Sep. 2010, pp. 137-140.

[36] K. Hirakawa and P. J. Wolfe, "Spatio-spectral color filter array design for optimal image recovery," in IEEE Transactions on Image Processing., vol. 17, no. 10, pp. 1876-1890, Oct. 2008

[37] L. Condat, "A new color filter array with optimal properties for noiseless and noisy color image acquisition," in IEEE Transactions on Image Processing., vol. 20, no. 8, pp. 2200-2210, Aug. 2011.

[38] Alleysson, D.; Susstrunk, S.; Herault, J., "Linear demosaicing inspired by the human visual system," in IEEE Transactions on Image Processing, vol.14, no.4, pp.439-449, April 2005
[39] T. Q. Pham, L. J. van Vliet, and K. Schutte, "Robust Fusion of Irregularly Sampled Data Using Adaptive Normalized Convolution," EURASIP Journal on Applied Signal Processing 2006, 2006. Article ID 83268, 12 pages.

[40] D. Robinson and P. Milanfar, "Statistical performance analysis of super-resolution," IEEE Transactions on Image Processing 15, pp. 1413-1428, June 2006

[41] Q.V. Le, A. Coates, B. Prochnow, and A.Y. Ng. ,"On optimization methods for deep learning."Learning, pages 265-272, 2011.

[42] Jia Li, Chenyan Bai, "Automatic Design of HighSensitivity Color Filter Arrays with Panchromatic Pixels" in IEEE Transactions on Image Processing, 2017

[43] J. Wang, C. Zhang, and P. Hao, "New color filter arrays of high light sensitivity and high demosaicking performance," in Proceedings of IEEE International Conference on Image Processing. IEEE, 2011, pp. 31533156

[44] L. Wang and G. Jeon, "Bayer Pattern CFA Demosaicking Based on Multi-Directional Weighted Interpolation and Guided Filter," in IEEE Signal Processing Letters, vol. 22, no. 11, pp. 2083-2087, Nov. 2015 